\title{
Detection of Significant Sets of Episodes in Event Sequences
}

\author{
Mikhail Atallah * \\ Purdue University \\ Department of Computer Sciences \\ mja@cs.purdue.edu \\ Robert Gwadera $^{\dagger}$ \\ Purdue University \\ Department of Computer Sciences \\ gwadera@cs.purdue.edu \\ Wojciech Szpankowski $\ddagger$ \\ Purdue University \\ Department of Computer Sciences \\ spa@cs.purdue.edu
}

\begin{abstract}
We present a method for a reliable detection of "unusual" sets of episodes in the form of many pattern sequences, scanned simultaneously for an occurrence as a subsequence in a large event stream within a window of size $w$. We also investigate the important special case of all permutations of the same sequence, which models the situation where the order of events in an episode does not matter, e.g., when events correspond to purchased market basket items. In order to build a reliable monitoring system we compare obtained measurements to a reference model which in our case is a probabilistic model (Bernoulli or Markov). We first present a precise analysis that leads to a construction of a threshold. The difficulties of carrying out a probabilistic analysis for an arbitrary set of patterns, stems from the possible simultaneous occurrence of many members of the set as subsequences in the same window, the fact that the different patterns typically do have common symbols or common subsequences or possibly common prefixes, and that they may have different lengths. We also report on extensive experimental results, carried out on the Wal-Mart transactions database, that show a remarkable agreement with our theoretical analysis. This paper is an extension of our previous work in [8] where we laid out foundation for the problem of the reliable detection of an "unusual" episodes,
\end{abstract}

*Portions of this author's work were supported by Grants EIA9903545, IIS-0219560, IIS-0312357, and IIS-0242421 from the National Science Foundation, Contract N00014-02-1-0364 from the Office of Naval Research, by sponsors of the Center for Education and Research in Information Assurance and Security, and by Purdue Discovery Park's eenterprise Center.

${ }^{\dagger}$ The work of this author was supported by the NSF Grant CCR0208709, and NIH grant R01 GM068959-01.

${ }^{\ddagger}$ The work of this author was supported by the NSF Grant CCR0208709, and AFOSR Grant FA 8655-04-1-3074. but did not consider more than one episode scanned simultaneously for an occurrence.

\section{Introduction}

Detecting subsequence patterns in event sequences is important in many applications, including intrusion detection, monitoring for suspicious activities, and molecular biology. Whether an observed pattern of activity is significant or not (i.e., whether it should be a cause for alarm) depends on how likely it is to occur fortuitously. A long enough sequence of observed events will almost certainly contain any subsequence, and setting thresholds for detecting significant patterns of activity is an important issue in a monitoring system.

In order to decide whether a particular sequence of events in the monitored event sequence is significant one must compare it to a reference model. In our work the reference model is a probabilistic model either generated by a memoryless (Bernoulli) source or a Markov source. The question is when is a certain number of occurrences of a particular subsequence in a monitored even sequence unlikely to be generated by the reference model (i.e., indicative of suspicious activity or statistically significant event)? A quantitative analysis of this question allows one to compute a threshold on-line while monitoring the event sequence in order to detect significant patterns. By knowing the most likely number of occurrences and the probability of deviating from it, we can compute a threshold such that the probability of missing real unusual activities is small. Such a quantitative analysis can also help to choose the size of the sliding window of observation. Finally even in a court case one cannot consider certain observed "bad" activity as a convincing evidence against somebody if that activity is 
quite likely to occur under the given circumstances. Therefore it is very important to quantify such probabilities and present a universal and reliable framework for analyzing a variety of event sources.

In [10] Mannila et al. introduced the problem of finding frequent episodes in event sequences, subject to observation-window constraint, where an episode was defined as a partially ordered collection of events, that can be represented as a directed acyclic graph. The paper [10] defined the frequency $f r(\alpha, s$, win $)$ of an episode $\alpha$ as the fraction of windows of length win in which the episode occurs in an event sequence $s$. Given a frequency threshold min_fr, [10] considered an episode to be frequent if $f r(\alpha, s$, win $) \geq \min _{-} f r$. In the framework of [10], our problem can be stated as follows. Given an episode $\alpha$, what window size win and what frequency threshold $m_{i n} f r$ should we choose to ensure that the discovered frequent episode is meaningful? Observe that for an appropriately low frequency $\min _{-} f r$ and large window size win the episode will certainly occur in the reference model. Our problem can also be stated as follows. Given a collection of frequent episodes $\mathcal{C}(w)$, discovered using the algorithm given in [10], what is the rank of the episodes with respect to their significance?

In our paper [8] the problem of the reliable detection of unusual episodes was investigated, where we considered an episode in the form of a single sequence occurring as an ordered subsequence of a large event stream within a window of a given fixed size. This kind of episode is called a "serial episode" in the terminology of [10], and we henceforth adopt this terminology. In [8] we proposed a method for reliable detection of significant episodes, where as a measure of significance we used $\Omega^{\exists}(n, w, m)$ the number of windows of length $w$ which contain at least one occurrence of serial episode $S$ of length $m$ as a subsequence in event sequence $T$ after $n$ shifts of the window. We proved that appropriately normalized $\Omega^{\exists}(n, w, m)$ has the Gaussian distribution, where the expected value $\mathbf{E}\left[\Omega^{\exists}(n, w, m)\right]=$ $n P^{\exists}(w, m)$ and $P^{\exists}(w, m)$ is the probability that a serial episode $S$ of length $m$ occurs at least once in a window of length $w$ in an event sequence $T$ over an alphabet $\mathcal{A}$. We also showed that the variance $\operatorname{Var}\left[\Omega^{\exists}(n, w, m)\right] \leq$ $c n\left[P^{\exists}(w, m)-\left(P^{\exists}(w, m)\right)^{2}\right]$ for $c>0$. Given a reference model (Bernoulli or Markov), and for a given probability $\beta(b)$, we presented the upper threshold for detecting significant episodes $\tau_{u}(w)=P^{\exists}(w, m)+\frac{b \sqrt{\operatorname{Var}\left[\Omega^{\exists}(n, w, m)\right]}}{n}$, where $P^{\exists}(w, m)$ and $\operatorname{Var}\left[\Omega^{\exists}(n, w, m)\right]$ depend on the probabilistic model and episode type. For a given probability $\beta(b)$ of the cumulative normal probability distribution function, we select $b$ such that $P\left(\frac{\Omega^{\exists}(n, w, m)}{n}>\tau_{u}(w)\right) \leq$ $\beta(b)$. That is, if one observes more than $\tau_{u}(w) \cdot n$ occurrences of windows with certain episodes, it is highly unlikely that such a number is generated by the reference source (i.e., its probability is smaller than $\beta(b)$ ). The quantity $\frac{\Omega^{\Xi}(n, w, m)}{n}$ corresponds to the frequency $\operatorname{fr}(S, T, w)$ [10] and is an estimator of $P^{\exists}(w, m)$ denoted $P_{e}^{\exists}(w, m)$.

While developing the formula for $P^{\exists}(w, m)$ we found a formula for the set of all distinct windows $\mathcal{W}^{\exists}(w, m)$ of length $w$ containing a serial episode $S$ of length $m$ at least once as a subsequence. The importance of $\mathcal{W}^{\exists}(w, m)$ stems from the fact that $P^{\exists}(w, m)=\sum_{x \in \mathcal{W}^{\exists}(w, m)} P(x)$ for the Markov model of an arbitrary order including 0-th order (Bernoulli), where $P(x)$ is the probability of $x$ as a string of symbols of length $w$ in a given model. The advantage of the Bernoulli model versus the first order Markov or higher is that for the Bernoulli model $P^{\exists}(w, m)$ can be computed efficiently exploiting the structure of $\mathcal{W}^{\exists}(w, m)$ and the fact that the model requires only $|\mathcal{A}|$ probabilities of symbols of the alphabet $\mathcal{A}$. Therefore in [8] we focused on the Bernoulli model for which we gave an efficient dynamic programming method for computing $P^{\exists}(w, m)$. Using generating functions and complex asymptotics we presented an asymptotic approximation of $P^{\exists}(w, m)$, which is of the form $P^{\exists}(w, m)=1-\Theta\left(\rho^{w}\right)$ for large $w$ and $0<\rho<1$. In experiments, we chose two apparently nonmemoryless sources (the English alphabet and the web access data) and showed that, even for these cases, $P^{\exists}(w, m)$ closely approximated the actual $P_{e}^{\exists}(w, m)$, which proved that the memoryless assumption did not limit the practical usefulness of the formula. We tested $\tau_{u}(w)$ by artificially injecting "bad" episodes into the monitored event sequence and observed that $\tau_{u}(w)$ did indeed provide a sharp detection of intentional (bad) episodes. Our paper [8] laid out foundations, but did not consider the case of detecting more than one serial episode simultaneously.

This paper builds on [8] by extending it to the case of an arbitrary number of serial episodes, monitored simultaneously for an occurrence, including the important special case of all permutations of the same serial episode, called a "parallel episode" in the terminology of [10]. The parallel episode case captures situations where the ordering of the events within the window of observation does not matter, e.g., the events correspond to basket items scanned by cashier. More formally, we analyze episodes in one of the following forms:

1. An arbitrary set of episodes $\mathbf{S}=\left\{S_{1}, S_{2}, \ldots, S_{|\mathbf{S}|}\right\}$ where every $S_{i}$ of length $m_{i}$ is a serial episode for $1 \leq$ $i \leq|\mathbf{S}|$ and by an occurrence of the set $\mathbf{S}$ we mean a logical $O R$ of occurrences of members of $\mathbf{S}$ within a window of size $w$.

2. Set of all distinct permutations of an episode $S=$ $S[1] S[2], \ldots S[m]$ of length $m$ (parallel episode), where by an occurrence we mean a logical $O R$ of occurrences of permutations of $S$. 
The reason we distinguish the parallel episode case is because we will take advantage of the structure of permutations of $S$ to design an efficient algorithm instead of representing the parallel episode as a set of serial episodes. Thus, the goal of the current paper is to quantify $\Omega^{\exists}\left(n, w, m_{1}, m_{2}, \ldots, m_{|\mathbf{S}|}\right)$ the number of windows containing at least one occurrence of $\mathbf{S}=\left\{S_{1}, S_{2}, \ldots, S_{|\mathbf{S}|}\right\}$ as a subsequence within a window of size $w$ in a event sequence $T$ over an alphabet $\mathcal{A}$. This analysis leads to a formula for the threshold for detecting significant sets of episodes. In the full version of this paper [3] we prove that $\Omega^{\exists}\left(n, w, m_{1}, m_{2}, \ldots, m_{|\mathbf{S}|}\right)$ is Gaussian. In order to compute $P^{\exists}\left(w, m_{1}, m_{2}, \ldots, m_{|\mathbf{S}|}\right)$ the probability that a window of length $w$ contains an occurrence of $\mathbf{S}$ we need a formula for $\mathcal{W}^{\exists}\left(w, m_{1}, m_{2}, \ldots, m_{|\mathbf{S}|}\right)$ the set of all windows of length $w$ containing an occurrence of $\mathbf{S}$ as a subsequence. However a considerable source of difficulty is the fact that $\mathcal{W}^{\exists}\left(w, m_{1}, m_{2}, \ldots, m_{|\mathbf{S}|}\right)$ for $|\mathbf{S}|>1$ is not equal to the enumeration of sets of $\mathcal{W}^{\exists}\left(w, m_{i}\right)$ for each $S_{i} \in \mathbf{S}$ because in general $\mathcal{W}^{\exists}\left(w, m_{i}\right) \cap \mathcal{W}^{\exists}\left(w, m_{j}\right) \neq \emptyset$ for $i \neq j$ where $i, j \leq|\mathbf{S}|$ and considering $\mathcal{W}^{\exists}\left(w, m_{i}\right)$ and $\mathcal{W}^{\exists}\left(w, m_{j}\right)$ independently would lead to a failure of the probabilistic analysis of $P^{\exists}\left(w, m_{1}, m_{2}, \ldots, m_{|\mathbf{S}|}\right)$ due to double counting of respective probabilistic events. To appreciate the difficulty of this extension, consider the much-simplified case when there are only two pattern sequences ( $\mathbf{S}=\left\{S_{1}, S_{2}\right\}$ ) and no symbol is common to $S_{1}$ and $S_{2}$. Even in this case the set of windows of length $w$ containing $S_{1}$ as a subsequence and the set of windows of length $w$ containing $S_{2}$ as a subsequence do have some elements in common, i.e., $\mathcal{W}^{\exists}\left(w, m_{1}\right) \cap \mathcal{W}^{\exists}\left(w, m_{2}\right) \neq \emptyset$ for appropriately large $w$. Add to this the fact that the different patterns typically do have common symbols or common subsequences or possibly common prefixes, that they may have different lengths, and the problem becomes fraught with nasty interactions that prevent any straightforward analytical solution to the case $|\mathbf{S}|>1$.

The main contribution of the current paper is a computational formula for $P^{\exists}\left(w, m_{1}, m_{2}, \ldots, m_{|\mathbf{S}|}\right)$ for and arbitrary set of episodes including the special case of the parallel episode. We provide a recurrence system for constructing $\mathcal{W}^{\exists}\left(w, m_{1}, m_{2}, \ldots, m_{|\mathbf{S}|}\right)$. Because the recurrence contains conditional statements representing interactions of symbols of members of $\mathbf{S}$ we cannot find a practical analytical solution to the recurrence. Therefore we propose an efficient algorithmic method for enumerating $\mathcal{W}^{\exists}\left(w, m_{1}, m_{2}, \ldots, m_{|\mathbf{S}|}\right)$ using recursion graphs, which leads to a formula for $P^{\exists}\left(w, m_{1}, m_{2}, \ldots, m_{|\mathbf{S}|}\right)$ for an arbitrary order of Markov model. However, we focus on Bernoulli model in this paper because of its compactness and efficiency. Our current work builds on [8] and provides the first probabilistic analysis that quantifies $\Omega^{\exists}\left(n, w, m_{1}, m_{2}, \ldots, m_{|\mathbf{S}|}\right)$ the number of windows of length $w$ containing at least one occurrence of $\mathbf{S}=$ $\left\{S_{1}, S_{2}, \ldots, S_{|\mathbf{S}|}\right\}$ as a subsequence. This analysis allows to compute the threshold for detecting significant sets of episodes scanned simultaneously for an occurrence. We also proposed a new $O(n \log (m))$ tree based algorithm for discovering parallel episodes, presented in the full version of the paper [3]. We applied our theoretical results by running an extensive series of experiments on real data. We used a part of Wal-Mart sales data for the years 1999 and 2000. We first show that our formulas for the probability closely approximate the experimental data. Then we demonstrate an application of the upper threshold by keeping inserting a given episode into random positions in the event sequence, until the episode gets detected as significant through our threshold mechanism (cf. Fig. 9).

The paper is organized as follows. In section 2 we present our main results containing theoretical foundation. Section 3 contains experimental results demonstrating applicability of the derived formulas. Proofs were omitted because of space limitations and are included in the full version of the paper in [3]. Interested readers can visit our on-line threshold calculator at http://www-cgi.cs . purdue.edu/cgi-bin/gwadera/demo.cgi for a demonstration of the reliable threshold computation.

\section{Main Results}

Given an alphabet $\mathcal{A}=\left\{a_{1}, a_{2}, \ldots, a_{|\mathcal{A}|}\right\}$ and a set of patterns $\mathbf{S}=\left\{S_{1}, S_{2}, \ldots S_{|\mathbf{S}|}\right\}$ where $S_{i}=$ $S_{i}[1] S_{i}[2] \ldots S_{i}\left[m_{i}\right]$ and $S_{i}[j] \in \mathcal{A}$ for $1 \leq i \leq|\mathbf{S}|$, we are interested in occurrences of members of $\mathbf{S}$ as a subsequence within a window of size $w$ in another sequence known as the event sequence $T=T[1] T[2] \ldots$.

We analyze the number of windows of length $w$ containing at least one occurrence of $\mathrm{S}$ when sliding the window along $n$ consecutive events in the event sequence $T$, where by an occurrence of $\mathbf{S}$ we mean a logical $O R$ of occurrences of $S_{1}, S_{2}, \ldots, S_{|\mathbf{S}|}$. We use $\Omega^{\exists}\left(n, w, m_{1}, m_{2}, \ldots, m_{|\mathbf{S}|}, \mathbf{S}, \mathcal{A}\right)$ to denote this number, that can range from 0 to $n$.

Notation: Throughout the paper, whenever $\mathcal{A}, \mathbf{S}$ or $m_{1}, m_{2}, \ldots, m_{|\mathbf{S}|}$ are implied and we do not reference them in our notations, we simplify our notations by dropping them accordingly using $\Omega^{\exists}(n, w), \mathcal{W}^{\exists}(w)$ and $P^{\exists}(w)$ instead. We also occasionally use index $m_{i}-k$ to mean "dropping the last k symbols of $S_{i}$ ", e.g., $P^{\exists}\left(w, m_{1}-k, m_{2}\right)$ implies a pattern that is the prefix of $S_{1}$ of length $m_{1}-k$ and that the second pattern is all of $S_{2}$.

Given a probabilistic model of the reference source (in this paper we use Bernoulli model with probabilities $P\left(a_{i}\right)$ for $\left.a_{i} \in \mathcal{A}, i=1,2, \ldots,|\mathcal{A}|\right)$, a frequent episode $\mathbf{S}, \Omega^{\exists}(n, w)$ and a probability $\beta(b)$ (e.g., $\beta(b)=10^{-5}$ ) we compute the upper threshold $\tau_{u}(w)$ for 
$P\left(\frac{\Omega^{\exists}(n, w)}{n} \geq \tau_{u}(w)\right) \leq \beta(b)$, using the equation system as follows

$$
\left\{\begin{array}{l}
\tau_{u}(w)=P^{\exists}(w)+\frac{b \sqrt{\operatorname{Var}\left[\Omega^{\exists}(n, w)\right]}}{n} \\
\beta(b)=\frac{1}{\sqrt{2 \pi}} \int_{\infty}^{b} e^{\frac{-t^{2}}{2}} d t
\end{array}\right.
$$

which follows from the fact that $\Omega^{\exists}(n, w)$ is Gaussian as we proved in [3]. Also, $P^{\exists}(w)$ and $\operatorname{Var}\left[\Omega^{\exists}(n, w)\right]$ depend on the episode type and the probabilistic model (Bernoulli or Markov). Once the threshold $\tau_{u}(w)$ is computed if $\frac{\Omega^{\exists}(n, w)}{n} \geq \tau_{u}(w)$ then the probability that the episode $\mathbf{S}$ is not significant is less than $\beta(b)$, i.e., the probability of an false alarm is less than $\beta(b)$. Given a collection of frequent episodes $\mathcal{C}(w)$ we can rank their significance using $\beta(b)$.

For the sake of the presentation we focus throughout the paper on the case where either $\mathbf{S}=\left\{S_{1}, S_{2}\right\}$, or $\mathbf{S}$ is the set corresponding to a parallel episode $S$ but our derivations will easily be seen to generalize to an arbitrary set of episodes $\mathbf{S}$.

\subsection{Analysis of $P^{\exists}(w)$}

Let $\mathcal{W}^{\exists}\left(w, m_{1}, m_{2}\right)$ be the set of all possible distinct windows of length $w$ containing $S_{1}$ or $S_{2}$ at least once as a subsequence then $P^{\exists}\left(w, m_{1}, m_{2}\right)=$ $\sum_{x \in \mathcal{W}^{\exists}\left(w, m_{1}, m_{2}\right)} P(x)$.

Because in the memoryless model $P(x)$ is a product of individual probabilities of symbols, to any recursive formula for $\mathcal{W}^{\exists}\left(w, m_{1}, m_{2}\right)$ there corresponds a similar formula for $P^{\exists}\left(w, m_{1}, m_{2}\right)$ (and vice-versa). We now show that $P^{\exists}\left(w, m_{1}, m_{2}\right)$ for the set $\mathcal{A}=\left\{S_{1}, S_{2}\right\}$ satisfies the following recurrence

$$
\left\{\begin{array}{l}
\text { if } S_{1}\left[m_{1}\right] \neq S_{2}\left[m_{2}\right] \text { then } \\
P^{\exists}\left(w, m_{1}, m_{2}\right)= \\
P\left(S_{1}\left[m_{1}\right]\right) P^{\exists}\left(w-1, m_{1}-1, m_{2}\right)+ \\
P\left(S_{2}\left[m_{2}\right]\right) P^{\exists}\left(w-1, m_{1}, m_{2}-1\right)+ \\
\left(1-P\left(S_{1}\left[m_{1}\right]\right)-P\left(S_{2}\left[m_{2}\right]\right)\right) P^{\exists}\left(w-1, m_{1}, m_{2}\right) \\
\text { for } w>0, m_{1}, m_{2}>0 \\
\text { if } S_{1}\left[m_{1}\right]=S_{2}\left[m_{2}\right] \quad \text { then } \\
P^{\exists}\left(w, m_{1}, m_{2}\right)= \\
P\left(S_{1}\left[m_{1}\right]\right) P^{\exists}\left(w-1, m_{1}-1, m_{2}-1\right)+ \\
\left(1-P\left(S_{1}\left[m_{1}\right]\right)\right) P^{\exists}\left(w-1, m_{1}, m_{2}\right) \\
\text { for } w>0, m_{1}, m_{2}>0 \\
P^{\exists}(w, 0,0)=1 \quad \text { for } \quad w>0 \\
P^{\exists}\left(0, m_{1}, m_{2}\right)=0 \quad \text { for } \quad m_{1}, m_{2}>0 \\
P^{\exists}\left(1, m_{1}, 0\right)=1 \quad \text { for } \quad m_{1}>0 \\
P^{\exists}\left(1,0, m_{2}\right)=1 \quad \text { for } \quad m_{2}>0 \\
P^{\exists}(0,0,0)=1 \quad \text { r } \quad
\end{array}\right.
$$

Indeed, consider a window of size $w$ containing $S_{1}$ or $S_{2}$ as a subsequence. Then depending on whether the last sym- bols of $S_{1}$ and $S_{2}$ are equal or not there are two cases. If $S_{1}\left[m_{1}\right] \neq S_{2}\left[m_{2}\right]$ then there are three cases: either $S_{1}\left[m_{1}\right]$ is the last symbol in the window giving the term $P\left(S_{1}\left[m_{1}\right]\right) P^{\exists}\left(w-1, m_{1}-1, m_{2}\right)$, or $S_{2}\left[m_{2}\right]$ is the last symbol in the window giving the term $P\left(S_{2}\left[m_{2}\right]\right) P^{\exists}(w-$ $\left.1, m_{1}, m_{2}-1\right)$, or none of the above which leads to the term $\left(1-P\left(S_{1}\left[m_{1}\right]\right)-P\left(S_{2}\left[m_{2}\right]\right)\right) P^{\exists}\left(w-1, m_{1}, m_{2}\right)$. If $S_{1}\left[m_{1}\right]=S_{2}\left[m_{2}\right]$ then there are two cases depending on whether the last symbol of the window is equal to $S_{1}\left[m_{1}\right]$ or not. From the above discussion it is clear that the shape of the "recursion graph" is determined by interactions between symbols in $S_{1}$ and $S_{2}$, i.e., whether their symbols at pairs of positions are equal or not. Therefore in order to find a solution to $P^{\exists}\left(w, m_{1}, m_{2}\right)$ we have to enumerate all pairs of indices $(i, j)$ such that $P^{\exists}(k, i, j)$ appears in the recursion tree (not all such pairs of indices qualify). This recursion graph is now described more formally (as stated earlier, in addition to depicting the recurrence, the graph also describes all elements of $\left.\mathcal{W}^{\exists}\left(w, m_{1}, m_{2}\right)\right)$.

Let $G(\mathbf{S})=(V, E)$ be an edge-labeled directed graph defined as follows. The vertex set $V$ is a subset of all the pairs $(i, j), 0 \leq i \leq m_{1}, 0 \leq j \leq m_{2}$. That subset, as well as $E$, are defined inductively as follows.

- $(0,0)$ is in $V$.

- If $(i, j)$ is in $V, i<m_{1}$, and $S_{1}[i+1] \neq S_{2}[j+1]$ then $(i+1, j)$ is also in $V$, and an edge from from $(i, j)$ to $(i+1, j)$ labeled $S_{1}[i+1]$ exists in $E$.

- If $(i, j)$ is in $V, j<m_{2}$, and $S_{1}[i+1] \neq S_{2}[j+1]$ then $(i, j+1)$ is also in $V$, and an edge from $(i, j)$ to $(i, j+1)$ labeled $S_{2}[j+1]$ exists in $E$.

- If $(i, j)$ is in $V, i<m_{1}$ and $j<m_{2}$, and $S_{1}[i+1]=$ $S_{2}[j+1]$ then $(i+1, j+1)$ is also in $V$, and an edge from $(i, j)$ to $(i+1, j+1)$ labeled $S_{1}[i+1]\left(=S_{2}[j+1]\right)$ exists in $E$.

- A self-loop from vertex $(i, j)$ to itself exists and has label equal to (i) $\mathcal{A}$ if $i=m_{1}$ or $j=m_{2}$, (ii) $\mathcal{A}-$ $\left\{S_{1}[i+1]\right\}-\left\{S_{2}[j+1]\right\}$ if $i<m_{1}$ and $j<m_{2}$.

The following observations, in which we do not count selfloops towards the in-degree and out-degree of a vertex, follow from the above definition of $G(\mathbf{S})$.

- The in-degree of vertex $(0,0)$ (start vertex) equals zero, the out-degree of vertices $\left(m_{1}, j\right),\left(i, m_{2}\right)$ for $i \leq m_{1}, j \leq m_{2}$ (end-vertices) equals zero.

- The in-degree and out-degree of every vertex $(i, j)$ is at most three; if $\mathbf{S}$ consisted of $|\mathbf{S}|>2$ serial episodes then the in-degree and out-degree of any vertex would be at most $|\mathbf{S}|+1$.

- $|V|=O\left(m_{1} m_{2}\right)$ and $|E|=O\left(|\mathbf{S}| m_{1} m_{2}\right)$. 
Let Edges(path) and Vertices(path) denote the sequence of consecutive edges and (respectively) vertices in any path, except that Vertices(path) does not include the last vertex on path (why this is so will become apparent below). In what follows, if vertex $(i, j)$ is in Vertices (path), then we use $n_{i, j}$ (path) to denote the number of times the selfloop at $(i, j)$ is used; if path is understood and there is no ambiguity, then we simply use $n_{i, j}$ rather than $n_{i, j}$ (path). Let $\mathcal{R}$ be the set of all distinct simple paths (i.e., without self-loops) from the start-vertex to any end-vertex. Now let $\mathcal{L}_{w}$ be the set of all distinct paths of length $w$, including self-loops, from the start-vertex to all end-vertices (that is, self-loops do count towards path length). Then we have

$$
\mathcal{W}^{\exists}\left(w, m_{1}, m_{2}\right)=\left\{\operatorname{Edges}(\text { path }): \text { path } \in \mathcal{L}_{w}\right\} .
$$

Examples of $G(\mathbf{S})$ are shown in Figure 1 and in Figure 2.

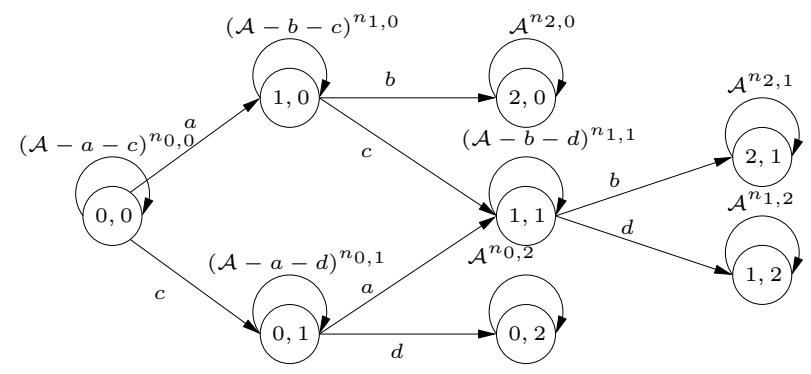

Figure 1. $G(\mathbf{S})$ for $\mathbf{S}=\{a b, c d\}$

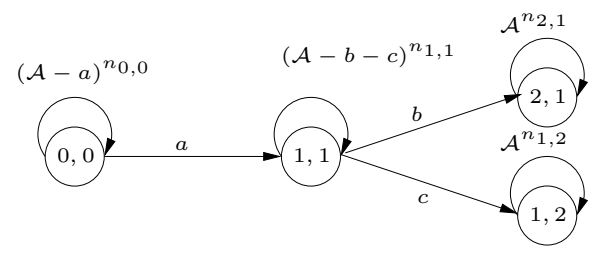

Figure 2. $G(\mathbf{S})$ for $\mathbf{S}=\{a b, a c\}$

Theorem 1 Consider a memoryless source with $P\left(S_{i}[k]\right)$ being the probability of generating the $k$-th symbol of $S_{i} \in$ $\mathbf{S}=\left\{S_{1}, S_{2}\right\}$. Let also

$$
P(\operatorname{Edges}(\text { path }))=\prod_{\text {edge } \in \text { Edges }(\text { path })} P(\text { label }(\text { edge })) .
$$

Then for $m_{1}, m_{2}$ and $w \geq m_{i}$ we have

$$
\begin{aligned}
& P^{\exists}\left(w, m_{1}, m_{2}\right)=\sum_{\text {path } \in \mathcal{R}} P(\text { Edges }(\text { path })) \sum_{g=0}^{w-\mid E d g e s(\text { path }) \mid} . \\
& \sum_{\sum n_{i, j}(\text { path })=g} \prod_{(i, j) \in \text { Vertices }(\text { path })} \\
& \left(1-P\left(\left\{S_{1}[i+1]\right\} \cup\left\{S_{2}[j+1]\right\}\right)\right)^{n_{i, j}(\text { path }) .}
\end{aligned}
$$

In our experiments, we implemented an efficient dynamic programming algorithm based on Theorem 1. In section 3.1.2 we present evidence how Theorem 1 works well on real data by comparing it to the estimate $P_{e}^{\exists}(w)=\frac{\Omega^{\exists}(n, w)}{n}$ given the actual $\Omega^{\exists}(n, w)$. We also solved $P^{\exists}(w)$ for an important special case when $\mathbf{S}$ consist of all permutations of one pattern $S$, which is the case of a parallel episode. Using Theorem 1 directly to design an algorithm in such an unordered case would be inefficient because we would then need to consider a graph having a disastrous $|V|=$ $O\left(m^{m !}\right)$.

In order to simplify the graph $G(\mathbf{S})$ that would result from all permutations, and bring its number of vertices down to a manageable size (quantified below), we exploit the structure of a set of all permutations to design a different graph. Notice that, for a parallel episode, every path in $\mathcal{R}$ is a permutation of symbols in $S$. In addition, the outdegree of a vertex is at most $m$ if all symbols of $S$ are different. Furthermore a transition from from $P^{\exists}(k, i, j, \ldots)$ to $P^{\exists}\left(k+1, i^{\prime}, j^{\prime}, \ldots\right)$ takes place for any symbol of $S$ not seen so far since the order of symbols does not matter. This observations allow us to introduce a variant of $G(\mathbf{S})$ called $G_{\|}(S)$.

Let $G_{\|}(S)=(V, E)$ be a directed edge-labeled graph defined as follows. The vertex set $V$ consist of submultisets of size $i=0,1, \ldots, m$ of the multiset of sorted symbols in $S$ denoted as $\{S[1], S[2], \ldots, S[m]\}$. We represent the submultisets equivalently as binary vectors of the form $\left(i_{1}, i_{2}, \ldots, i_{m}\right)$, where $i_{j}=1$ if the vertex contains symbol $S\left[i_{j}\right]$ in its submultiset or $i_{j}=0$ otherwise. $V$ and $E$ can be defined inductively as follows.

- $(0,0, \ldots, 0)$ and $(1,1, \ldots, 1)$ are in $V$.

- If $\left(i_{1}, i_{2}, \ldots, i_{m}\right)$ is $V$ and $\sum_{j=1}^{m} i_{j}<m$ then $\left(i_{1}^{\prime}, i_{2}^{\prime}, \ldots, i_{m}^{\prime}\right)$ is in $V$ if $\sum_{j=1}^{m} i_{j}^{\prime}=\sum_{j=1}^{m} i_{j}+1$ and an edge with label equal to $\left\{i_{1}^{\prime} \cdot S[1], i_{2}^{\prime} \cdot S[2], \ldots, i_{m}^{\prime}\right.$. $S[m]\}-$

$\left\{i_{1} \cdot S[1], i_{2} \cdot S[2], \ldots, i_{m} \cdot S[m]\right\}$ exists in $E$.

- A self-loop from vertex $\left(i_{1}, i_{2}, \ldots, i_{m}\right)$ to itself exists and has label equal to (i) $\mathcal{A}$ for $(1,1, \ldots, 1)$, (ii) $\mathcal{A}$ $\bigcup_{i_{j}=0}\left\{S\left[i_{j}\right]\right\}$ otherwise.

The following observations, in which we do not count selfloops towards the in-degree and out-degree of a vertex, follow from the above definition of $G_{\|}(S)$.

- The in-degree of the start-vertex $(0,0, \ldots, 0)$ equals zero, the out-degree of the end-vertex $(1,1, \ldots, 1)$ equals zero. The in-degree and out-degree of every vertex is at most $m$.

- $|V|=O\left(2^{m}\right)$ and $|E|=O(|V| m)$. 
Examples of $G_{\|}(S)$ are shown in Figure 3 and in Figure 4. The next theorem is an adoption of Theorem 1 to the case of a parallel episode.

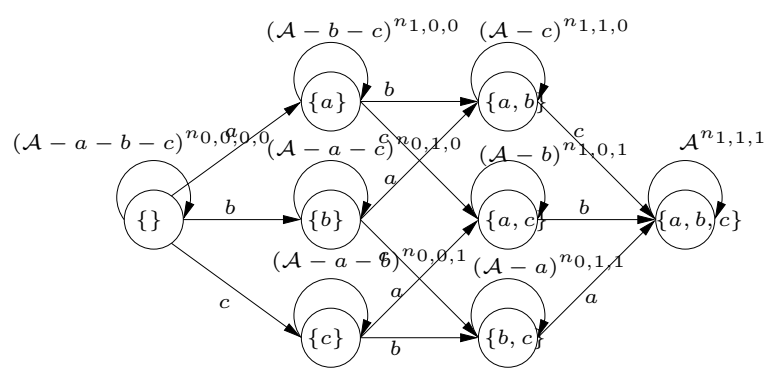

Figure 3. $G_{\|}(S)$ for $S=a b c$ and $\mathcal{A}=\{a, b, c, d\}$

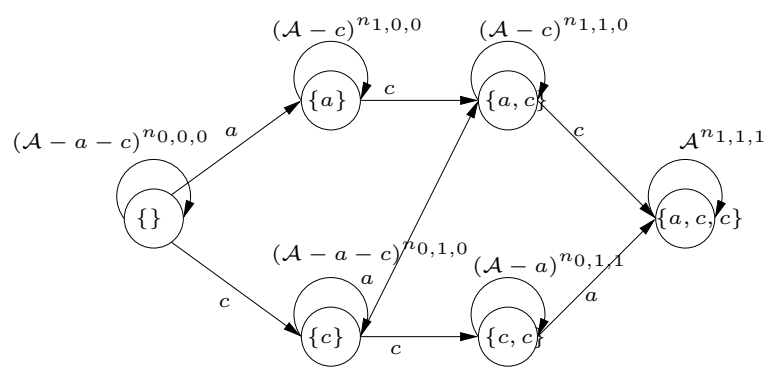

Figure 4. $G_{\|}(S)$ for $S=a c c$ and $\mathcal{A}=\{a, b, c, d\}$

Theorem 2 Consider a memoryless source with $P(S[k])$ being the probability of generating the $k$-th symbol of $S$ where $S$ is a parallel episode with

$$
P(S)=\prod_{i=1}^{m} P(S[i])
$$

then for all $m$ and $w \geq m$ we have

$$
\begin{aligned}
& P^{\exists}(w)=\sum_{\text {path } \in \mathcal{R}} P(S) \sum_{g=0}^{w-m} \\
& \sum_{\sum n_{i_{1}, i_{2}, \ldots, i_{m}}(\text { path })=g} \prod_{\left(i_{1}, i_{2}, \ldots, i_{m}\right) \in \operatorname{Vertices}(\text { path })} . \\
& \left(1-P\left(\bigcup_{i_{j}=0}\left\{S\left[i_{j}\right]\right\}\right)\right)^{n_{i_{1}, i_{2}, \ldots, i_{m}}(\text { path })} .
\end{aligned}
$$

In our experiments we implemented an efficient dynamic programming algorithm based on Theorem 2 for computing $P^{\exists}(w)$. In section 3.1.1 we presented how Theorem 2 works well on real data where the formula for $P^{\exists}(w)$ agrees with $P_{e}^{\exists}(w)$ on the Wal-Mart transactions.

\section{Experiments}

The purpose of our experiments was to test applicability of the analytical results for real sources. Therefore we selected Wal-Mart data available on the departmental Oracle server in the Department of Computer Sciences, Purdue University. The database contains part of Wal-Mart sales data for the years 1999 and 2000 in 135 stores. We selected one of the stores, one category of items of cardinality $35(|\mathcal{A}|=35)$ and extracted 9.66 million records from table Item_Scan, sorted by scan time. We divided our sources into training sets and testing sets. Training sets are data sets, which we consider to constitute the reference source. We used the first 9.56 million records as a training set to compute $P\left(a_{i}\right)$ for $a_{i} \in \mathcal{A}, i=1,2, \ldots,|\mathcal{A}|$ for the Bernoulli reference source. Once the probability model of the reference source has been built, we can start monitoring unknown data called testing data. In section 3.1 we tested how well the formulas for $P^{\exists}(w)$ worked on the WalMart data by comparing $P_{e}^{\exists}(w)=\frac{\Omega^{\exists}(n, w)}{n}$ to the computed $P^{\exists}(w)$ for different values of $w$. We used the following error metric $d=\left[\frac{1}{r} \sum_{i=1}^{r} \frac{\left|P_{e}^{\exists}\left(w_{i}\right)-P^{\exists}\left(w_{i}\right)\right|}{P_{e}^{\exists}\left(w_{i}\right)}\right] 100 \%$ where $w_{1}<w_{2}<\ldots w_{r}$ are the tested window sizes. In section 3.2 we tested the detection properties of the threshold $\tau_{u}(w)$ as a function of the window length $w$. All our algorithms have been implemented in $\mathrm{C}++$ and run under Linux operating system.

\subsection{Estimation of $P^{\exists}(w)$}

In all experiments in this section we used the same testing source of length $n=10^{5}$ events.

\subsubsection{The case of a parallel episode $S$}

We set $S=\left\{i t_{0}, i t_{4}, i t_{5}, i t_{6}, i t_{9}, i t_{10}, i t_{17}\right\}$ and then ran the tree based detection algorithm [3] for finding $\Omega\left(10^{5}, w\right)$ for $w \in[10,180]$ and compared $P_{e}(w)$ to the analytically computed $P^{\exists}(w)$ using our algorithm based on Theorem 2 . The results are shown in Figure 5, which indicate an exceptionally close fit between $P^{\exists}(w)$ and $P_{e}^{\exists}(w)$ with the difference $d$ of order $2 \%$. The results confirm our expectations that the Bernoulli model and parallel episode models well sources as the Wal-Mart item scans where the source seems to generate events independently.

\subsubsection{The case of a set of two serial episodes}

We set $S_{1}=\left\{i t_{0}, i t_{4}, i t_{5}, i t_{6}, i t_{9}, i t_{10}, i t_{17}\right\}$ and $S_{2}=$ $\left\{i t_{0}, i t_{6}, i t_{5}, i t_{4}, i t_{10}, i t_{9}, i t_{17}\right\}$ where $S_{2}$ is a permuted version of $S_{1}$. This case reflects a situation when a pattern of interest is only partially restricted and the serial case is too restrictive but the parallel case too relaxed. We ran an algorithm for finding $\Omega\left(10^{5}, w\right)$ for $\mathbf{S}$ for $w \in[10,180]$. Then compared $P_{e}(w)$ to the analytically computed $P^{\exists}(w)$ using our algorithm based on Theorem 1 . The results are shown in Figure 6, which indicate a very close fit between $P^{\exists}(w)$ 


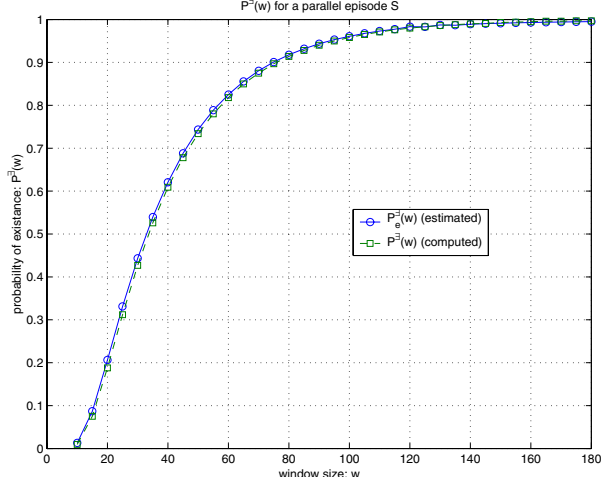

Figure 5. $P_{e}^{\exists}(w)=\frac{\Omega^{\exists}(n, w)}{n}$ and $P^{\exists}(w)$ for a parallel episode $S$, using Wal-Mart data

and $P_{e}^{\exists}(w)$ with $d$ of order $13 \%$ but not so close as in the parallel case $(d=2 \%)$. The reason may be the fact that this set of episodes is too restricted comparing to the parallel episode given the unordered nature of the item scans.

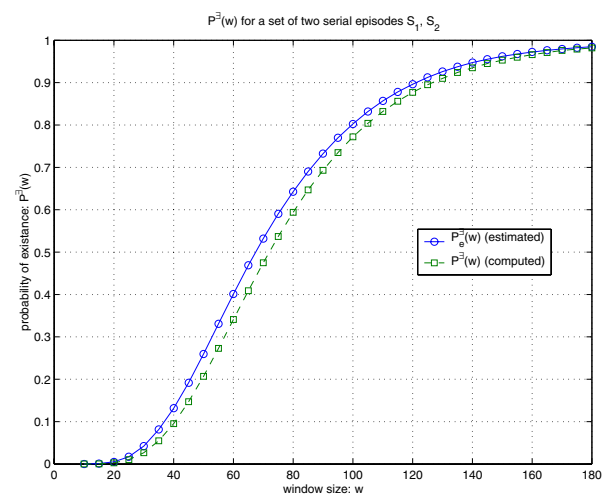

Figure 6. $P_{e}^{\exists}(w)=\frac{\Omega^{\exists}(n, w)}{n}$ and $P^{\exists}(w)$ for a set $\mathbf{S}=\left\{S_{1}, S_{2}\right\}$ of serial episodes, using Wal-Mart data

\subsubsection{Comparison of the three cases: parallel, two se- rial and one serial}

In this experiment we investigate the relationship between the formulas for $P(w)$ and the experimental $P_{e}(w)$ for the episode $S$ in the three cases: parallel, partially ordered ( $S_{2}$ is a permutation of $S_{1}$ ) and serial (investigated in [8]). For the first two cases we use the results obtained in the previous experiments. For the third case we ran an algorithm for discovering a serial episode in the event sequence for $w \in[10,180]$ as in the previous experiment to create $\Omega\left(10^{5}, w\right)$ at the same points. The results for $P^{\exists}(w)$ are shown in Figures 7 and the corresponding estimates are shown in Figure 8. The figures clearly indicate that the serial and parallel cases of an episode $S$ establish the lower and upper bound on the probability of existence of $S$ in window of size $w$.

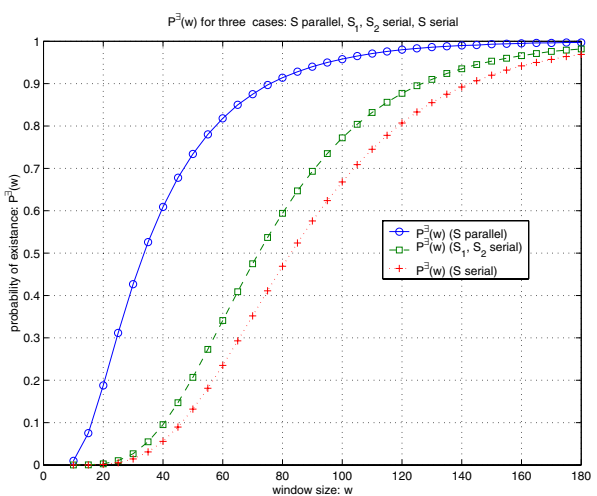

Figure 7. $P^{\exists}(w)$ for three cases: $S$ parallel, $\left\{S_{1}, S_{2}\right\}$ serial and $S$ serial, using Wal-Mart data

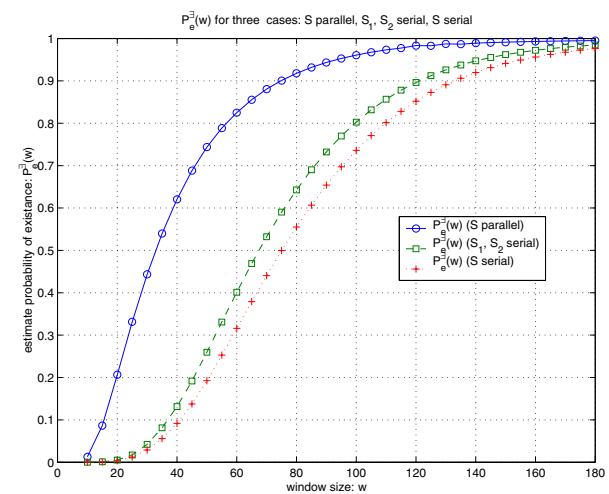

Figure 8. $P_{e}^{\exists}(w)=\frac{\Omega^{\exists}(n, w)}{n}$ for three cases: $S$ parallel, $\left\{S_{1}, S_{2}\right\}$ serial and $S$ serial, using Wal-Mart data

\subsection{Threshold $\tau_{u}(w)$}

This experiment demonstrates an application of the upper threshold $\tau_{u}(w)$ for detecting a significant number of occurrences of a parallel episodes $S$. It also shows the relationship between $\tau_{u}(w)$ and $w$ in detecting unusual episodes. We set $S=\left\{i t_{8}, i t_{12}, i t_{14}, i t_{15}, i t_{19}, i t_{20}, i t_{26}\right\}$ and consider the parallel case of $S$. We selected a part of the scans of length $n=50000$ as the test source. We compute the threshold $\tau_{u}(w)=P^{\exists}(w)+b \frac{\sqrt{\operatorname{Var}\left[\Omega^{\exists}(n, w)\right]}}{n}$ for 
$\beta(b)=10^{-6}$ for which we obtained $b=4.26$ using an algorithm for computing the inverse of $\beta(b)$. We repeated the threshold computation for three values of $w=40,30,25$ for the same episode $S$. We simulated an attack by keeping inserting the episode $S$ as a string into the testing source until we exceeded the threshold. We normalized the number of insertion by $n$. Figure 9 presents results. We conclude that the if $w$ increases then the number of attacks causing $\frac{\Omega^{\exists}(n, w)}{n}$ to rise above the $\tau_{u}(w)$ increases exponentially which is caused by the exponential growth of $P^{\exists}(w)$ in the formula for $\tau_{u}(w)$.

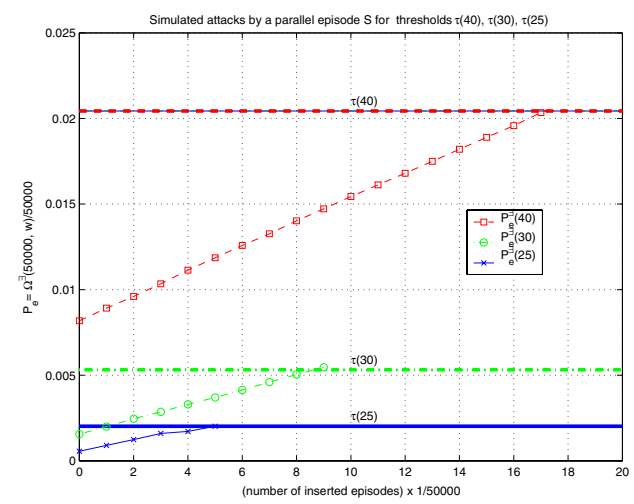

Figure 9. The upper threshold $\tau_{u}(w)$ as a function of $w$ for a parallel episode $S$, using the Wal-Mart database

\section{Conclusions}

We presented the exact formulas for the probability of existence $P^{\exists}(w)$, for an arbitrary set of serial episodes $\mathbf{S}$ including the case of a parallel episode $S$. Using the formulas we showed how to compute the upper threshold $\tau_{u}(w)$ to measure significance of $\mathbf{S}$. Since we adopted a computational probability approach it is valid for the Markov model of any order. The choice of the 0-the order (Bernoulli) was dictated only by its compactness and suitability to implementation through efficient dynamic programming algorithms. However in experiments on Wal-Mart transactions we showed that formulas for the Bernoulli model very closely approximated the real life data. Realization of Markov model of order higher then 0 would require computation of $P^{\exists}(w)$ using conditional probabilities of the respective order.

\section{Acknowledgment}

The authors are very grateful to Prof. Chris Clifton for many valuable remarks and encouragements.

\section{References}

[1] A. Aho and M. Corasick (1975), Efficient String Matching: An Aid to Bibliographic Search Programming Techniques.

[2] A. Apostolico and M. Atallah (2002), Compact Recognizers of Episode Sequences, Information and Computation, 174, 180-192.

[3] M. Atallah, R. Gwadera and W. Szpankowski. Detection of significant sets of episodes in event sequences. http://www.cs.purdue.edu/ homes/gwadera/icdm2full.ps.

[4] P. Billingsley (1986), Probability and measure, John Wiley, New York.

[5] L. Boasson, P. Sequels, I. Guessarian, and Y. Matiyasevich (1999), Window-Accumulated Subsequence Matching Problem is Linear, Proc. PODS, 327-336.

[6] G. Das, R. Fleischer, L. G asieniec, D. Gunopulos, and J. Kärkkäinen (1997), Episode Matching, In Combinatorial Pattern Matching, 8th Annual Symposium, Lecture Notes in Computer Science vol. 1264, 12-27.

[7] P. Flajolet, Y. Guivarc'h, W. Szpankowski, and B. Vallée (2001), Hidden Pattern Statistics, ICALP 2001, Crete, Greece, LNCS 2076, 152-165.

[8] R. Gwadera, M. Atallah, and W. Szpankowski. Reliable detection of episodes in event sequences. In Third IEEE International Conference on Data Mining, pages 67-74, Melbourne, Florida.

[9] J. Han, J. Pei, Y. Yin, R. Mao, Mining Frequent Patterns without Candidate Generation: A FrequentPattern Tree Approach, Data Mining and Knowledge Discovery, 8, 53-87, 2004

[10] H. Mannila, H. Toivonen, and A. Verkamo (1997), Discovery of frequent episodes in event sequences Data Mining and Knowledge Discovery, 1(3), 241258.

[11] M. Régnier and W. Szpankowski (1998), On pattern frequency occurrences in a Markovian sequence $\mathrm{Al}$ gorithmica, 22, 631-649.

[12] W. Szpankowski (2001), Average Case Analysis of Algorithms on Sequence, John Wiley, New York. 